\title{
Liter per Hour per Microgram per Kilogram
}

National Cancer Institute

\section{Source}

National Cancer Institute. Liter per Hour per Microgram per Kilogram. NCI Thesaurus.

Code C120746.

A unit of concentration equal to liter per hour divided by microgram per kilog ram. 